\title{
Erratum: Superboost transitions, refraction memory and super-Lorentz charge algebra
}

\author{
Geoffrey Compère, Adrien Fiorucci and Romain Ruzziconi \\ Université Libre de Bruxelles and International Solvay Institutes, \\ CP 231, B-1050 Brussels, Belgium \\ E-mail: gcompere@ulb.ac.be, afiorucc@ulb.ac.be, rruzzico@ulb.ac.be
}

ERRATUM TO: JHEP11(2018)200

ARXIV EPRINT: 1810.00377

\section{List of corrections}

Hereby, we bring to your attention the following typos and mistakes in [1].

After correcting one algebraic mistake and one typo, equation (5.31) must read as

$$
\begin{aligned}
\varnothing \bar{H}_{\xi}^{(0)}=\frac{1}{16 \pi G} \oint \mathrm{d}^{2} \Omega[ & \left.4 f M+2 Y^{A} N_{A}+\frac{1}{16} Y^{A} \partial_{A}\left(C_{B C} C^{B C}\right)\right] \\
+\frac{1}{16 \pi G} \oint \mathrm{d}^{2} \Omega & {\left[\frac{1}{2} f\left(N^{A B}+\frac{1}{2} q^{A B} \stackrel{\circ}{R}\right) \delta C_{A B}-2 \partial_{(A} f \dot{\circ}_{B)} \delta q^{A B}\right.} \\
& \left.-f D_{(A} \stackrel{\circ}{U}_{B)} \delta q^{A B}-\frac{1}{4} D_{C} D^{C} f C_{A B} \delta q^{A B}\right] .
\end{aligned}
$$

Equation (5.32) is the modification of the surface charge codimension 2-form $\boldsymbol{k}_{\xi}[g, \delta g]$ under the incorporation of a covariant Iyer-Wald ambiguity $\boldsymbol{Y}[g, \delta g]$. Its proof involves Cartan's magic formula acting on $\boldsymbol{Y}$. Here however, $\boldsymbol{Y}$ is not proven to be covariant in terms of the bulk metric alone and therefore that identity cannot be used. As a consequence, although (5.33)-(5.36) are correct, the comment just below (5.36) must be deleted.

Instead, the boundary counterterm $\boldsymbol{Y}$ removes all linear in $r$ divergences without affecting the finite pieces by construction. According to (1.1), the finite charge in (5.37) must read as

$$
\not H_{\xi}=\varnothing \bar{H}_{\xi}^{(0)} .
$$


We checked that the $u$ derivative of this charge is equal to the symplectic flux $\oint \mathrm{d}^{2} \Omega \omega^{r}\left[g, \delta_{\xi} g, \delta g\right]$ where $\omega^{r}$ is defined in (5.26). The surface charge expression is therefore now correct. (5.38) is therefore incorrect and the non-integrable part reads as

$$
\begin{aligned}
\Xi_{\xi}[g, \delta g]=\frac{1}{16 \pi G} \oint \mathrm{d}^{2} \Omega[ & \frac{1}{2} f\left(N^{A B}+\frac{1}{2} q^{A B} \stackrel{\circ}{R}\right) \delta C_{A B}-2 \partial_{(A} f \stackrel{\circ}{U}_{B)} \delta q^{A B} \\
& \left.-f D_{(A} \stackrel{\circ}{U}_{B)} \delta q^{A B}-\frac{1}{4} D_{C} D^{C} f C_{A B} \delta q^{A B}\right] .
\end{aligned}
$$

Due to the modification of the surface charge (5.37), the algebra of charges, described in (5.68)-(5.70), closes under the modified bracket. (5.68) should be replaced by

$$
\delta_{\xi_{1}} H_{\xi_{2}}[g]+\Xi_{\xi_{1}}\left[g, \delta_{\xi_{2}} g\right]=H_{\left[\xi_{2}, \xi_{1}\right]}[g]+\mathcal{K}_{\xi_{1}, \xi_{2}}[g] .
$$

There is no anomalous term and (5.70) should be removed. Equation (5.69) should be replaced by

$$
\begin{aligned}
\Xi_{\xi_{1}}\left[g, \delta_{\xi_{2}} g\right]=\frac{1}{16 \pi G} \oint \mathrm{d}^{2} \Omega & {\left[\frac{1}{2} f_{1}\left(N^{A B}+\frac{1}{2} q^{A B} \stackrel{\circ}{R}\right) \delta_{\xi_{2}} C_{A B}-2 \partial_{(A} f_{1}{\stackrel{\circ}{U_{B}}}_{B} \delta_{\xi_{2}} q^{A B}\right.} \\
& \left.-f_{1} D_{(A} \stackrel{\circ}{U}_{B)} \delta_{\xi_{2}} q^{A B}-\frac{1}{4} D_{C} D^{C} f_{1} C_{A B} \delta_{\xi_{2}} q^{A B}\right]-\delta_{\xi_{2}} \Delta H_{\xi_{1}} .
\end{aligned}
$$

The comments under (5.72) must be modified as follows: "The charge algebra (5.68) closes under the modified bracket introduced in [2]. Even staying...". Accordingly, the last sentence of the introduction before the Note added should read as "We finally show that the generalized BMS charges obey the algebra under the modified Dirac bracket introduced in $[2]$ ".

The technical restriction (2.4) can be better reformulated as the boundary condition $\sqrt{q}=\sqrt{\bar{q}}$ where $\partial_{u} \bar{q}=0$ and $\delta \bar{q}=0$. Then $\partial_{u} q_{A B}=0$ follows from Einstein's equations. The residual diffeomorphisms (2.10) preserve the condition $\sqrt{q}=\sqrt{\bar{q}}$. In the introduction of section 2.1., $r$ is a parameter along the null geodesic congruence which is not necessarily affine. The right-hand side of (2.23) needs to include the additional term $+\frac{1}{8} D_{A} D_{B} D_{C} Y^{C} C^{A B}$. In addition, note that all $\int \mathrm{d}^{2} \Omega$ should read as $\oint \mathrm{d}^{2} \Omega, C_{A B}=2\left(u^{-1}\right)$ in footnotes 4 and 5 should read as $C_{A B}=o\left(u^{-1}\right)$; the subscripts $c$ in section 3.1 are better written $C$ in order to avoid confusion with the indices $a, b, c$; all subscripts (0) in section 5.3 are better written as (fin) in order to match the notation of section 5.4. The integration variable in (5.10) should be $\mathrm{d} u \mathrm{~d} r \mathrm{~d}^{2} x$ while $\mathrm{d}^{2} \Omega$ in (5.21) is $\sqrt{q} \mathrm{~d}^{2} x$; the sign of the $r$ divergent part in (5.24) should be the opposite; in the first term of (5.26), the $q_{A B}$ should be $q^{A B}$; there is a "hat" missing on $N_{A}^{\mathrm{vac}}$ in (5.65). Finally, the "spin effect" in the box in figure 2 should better be called "spin and center-of-mass memory".

Open Access. This article is distributed under the terms of the Creative Commons Attribution License (CC-BY 4.0), which permits any use, distribution and reproduction in any medium, provided the original author(s) and source are credited. 


\section{References}

[1] G. Compère, A. Fiorucci and R. Ruzziconi, Superboost transitions, refraction memory and super-Lorentz charge algebra, JHEP 11 (2018) 200 [arXiv:1810.00377] [INSPIRE].

[2] G. Barnich and C. Troessaert, BMS charge algebra, JHEP 12 (2011) 105 [arXiv:1106.0213] [INSPIRE]. 